\title{
Synthetic cathione and cannabinoid toxicity and treatment
}

\section{Introduction}

Designer drugs, such as synthetic cathinones ${ }^{1}$ or bath salts, are synthetic derivatives of illegal or legal controlled substances created for recreational highs and to evade legal ramifications. There are many variations of the substance known as "bath salts". ${ }^{2}$ Bath salts are advertised as a cheap, legal substitute for cocaine. The side effect profile for bath salts include paranoia, aggressive violent behavior, hallucinations,${ }^{3}$ delusions, suicidal thoughts, seizure, panic attacks, increase blood pressure and heart rate, chest pain, fever, and nausea or vomiting. The two common ingredients found in bath salts are 3,4-methylene-dioxyprovalerone (MDPV) ${ }^{4}$ and 4-methylcathinone (mephedrone). MDPV is a dopamine and norepinephrine reuptake inhibitor that acts as a stimulant. Mephedrone is thought to be a monoamine reuptake inhibitor. The mechanism of action includes an increase in serotonin, norepinephrine, and dopamine levels. Mephedrone ${ }^{5}$ is a derivative of phenethylamine, which is the active ingredient in the African plant khat. Khat is similar to amphetamine and is associated with a history of abuse.

Federal legislation was passed in September of 2011 to restrict the possession or distribution of any substance containing MDPV, methadone, or methylone. However, synthetic stimulants are difficult to regulate. These substances are easily manipulated into other compounds as soon as legislation banning a specific compound is passed. The drug class of phenethylamines, which these compounds belong to, is unable to be banned outright because this class also contains FDA-approved medications, such as bupropion, dopamine, and epinephrine. The Federal Analog Act (21 USC 813) was passed in an effort to avoid the need for legislation to be passed on each individual analog. However, the Analog Act only applies to products meant for human consumption. Bath salts are labeled "not for human consumption" as a way around this law.

The American Association of Poison Control Centers ${ }^{6}$ report the number of calls related to bath salts increasing from 304 in 2010 to 2,655 in 2012. The patients' ages ranged from 1day to 61years, with a median age of 29.2 years. The clinical effects were primarily neurological or cardiovascular. In a recent retrospective review of hospital visits reported to poison control centers, 1,633 patients with reported bath salt toxicity were examined. Death resulted in $0.6 \%$ of patients. The reported reasons for death include suicide, serotonin syndrome, fever, and seizures. Major outcomes, defined as life threatening or resulting in significant disability or disfigurement, occurred in $15.5 \%$ of patients. The route of administration and age of the patient have been shown as factors influencing the occurrence of major outcomes or death. The injection of bath salts was predicted to have more serious effects than snorting bath salts. There was no difference in major outcomes or death between oral ingestion and injection. Patients aged 47 years or greater also had a higher risk of major outcomes than the younger populations. This older population also was shown to have higher rates of injection and co-ingestions, as well. Co-ingestions of other substances presenting with bath salt toxicities have a relatively low occurrence as compared to other
Volume 4 Issue 6 - 2016

\author{
Leah Sheffer \\ University of Florida/Pipeline Rx, USA
}

Correspondence: Leah Sheffer, University of Florida/ Pipeline Rx, 619 S Surrey Lane Carbondale, IL 6290I, USA, Tel 6183033626,Email leahcsheffer@gmail.com

Received: April 27, 2015 | Published: November 03, 2016

substances of abuse, around $26 \%$. The most common co-ingestion was with opioids in $6.2 \%$ of the reported cases from November 2011 to November 2012, followed by marijuana in $5.4 \%$ of reported case. This data suggests that the majority of bath salt abusers are seeking "legal highs".

The treatment of the sympathomimetic toxidrome associated with bath salts should start with the administration of benzodiazepines. It is reported that benzodiazepines are used in about $60 \%$ of patients. Amphetamine-induced seizures typically result from an imbalance of excitatory and inhibitory neurotransmitters that do not originate in a specific location of the brain. Amphetamine-induced seizures respond to benzodiazepines, which also act to sedate the patient for the safety of the healthcare team. Phenytoin and Fosphenytoin should not be used as they work by isolating the focal point of a seizure and can induce arrhythmias. Lorazepam and Diazepam are the preferred agents via the intravenous route. Sedation is also a common treatment strategy used in this patient population. Sedative agents commonly used include propofol, barbiturates, and antipsychotics. Almost 10\% of patients presenting with bath salt intoxication require ventilation. Psychotic symptoms typically resolve within 12 hours to 3 days of admission, although one patient remained symptomatic for 11 days. Caution must be used when administering anti-psychotic medications for drug-induced psychoses, as they may lower the seizure threshold.

Aggressive cooling measures should be initiated if the patient presents with a fever. If the patient is hyponatremic, hypertonic saline and water restriction should be prescribed. The goal rate of therapy for sodium correction should be 1 to $2 \mathrm{mmol} / \mathrm{L} / \mathrm{hr}$, not to exceed the maximum correction of $10 \mathrm{mmol} / \mathrm{L} / 24 \mathrm{hr}$ as to avoid osmotic myelinolysis. Hypertonic saline use should be reserved for patients presenting with hyponatremia with neurologic manifestations. The sodium correction rate in these patients is more aggressive because of the high incidence of seizures. If the patient presents with acute coronary syndrome or symptoms, consider nitroglycerin, morphine, and antiplatelet medications as treatment options. However, the use of beta-blockers is contraindicated, as they may have unpredictable effects on the blood pressure and heart rate. Co-administration of a 
beta-blocker with a $\mathrm{CYP}_{2} \mathrm{D}_{6}$ inhibitor, such as cocaine ${ }^{8}$ or a potential component of bath salts, may result in a 2 - to 8 -fold increase the serum concentration of the beta-blocker.

Ecstasy is another drug of abuse gaining in popularity. Most of the first time users of ecstasy, also known as 3,4-methylenedioxymethamphetamine (MDMA), ${ }^{9}$ are referred to the emergency departments and maintained under surveillance for several hours prior to discharge. It is estimated that $9 \%$ of patients presenting to the emergency departments with acute ecstasy intoxications are transferred to the intensive care units. Typically, these patients are more commonly chronic abusers. MDMA's structure is similar to epinephrine and norepinephrine. It causes central nervous stimulation, tachycardia, hypertension, and dilated pupils. Patients may also present with seizures, myocardial infarction, or coma. MDMA is unique from other amphetamines in that it has more potent serotonergic properties. MDMA works by increasing the release and inhibiting the reuptake of dopamine, norepinephrine, and serotonin into the postsynaptic neurons. In addition, MDMA also prevents the destruction of these neurotransmitters through inhibition of monoamine oxidase. Chronic use is associated with depression, impaired cognitive function, and abnormal patterns of serotonergic function on PET scanning. These effects may be due to an alteration of serotonergic metabolism leading to serotonin depletion in the central nervous system. The psychedelic properties seen in MDMA intoxication are likely mediated by dopamine and serotonin. Possible complications of MDMA intoxication include hyperthermia, hyponatremia, acute myocardial infarction, hepatic failure, renal failure, disseminated intravascular coagulation, and non-cardiogenic pulmonary edema. The hepatic and renal failure may recover without medical interventions. No relationship between the severity of liver failure and neither the amount of frequency of MDMA ingested has been established.

Toxicology reports are recommended, although not always reliable when negative results are obtained. MDMA has a short half-life and is often detectable in blood for only 6hours after ingestion. The most common adverse effect of MDMA requiring a therapeutic intervention is hyperthermia. Life-threatening fever, as high as 43.3 , or $109^{\circ} \mathrm{f}$, has been reported after MDMA use. Historically, treatment has consisted of hydration to reduce the body temperature. Hydration therapy may help to prevent Hypovolemia, rhabdomyolysis, and other renal complications. However, hydration can contribute to the development and progression of hyponatremia. Therapeutic normothermia or other methods of aggressively cooling the body are recommended early in therapy. Hyponatremia, a major cause of morbidity and mortality secondary to MDMA intoxication, is often seen within hours of drug ingestion. Patients with MDMA-induced hyponatremia commonly present with serum sodium levels between 115 and $125 \mathrm{mmol} / \mathrm{L}$, although a level of $101 \mathrm{mmol} / \mathrm{L}$ was reported. The exact mechanism of hyponatremia is unknown. It is hypothesized that since MDMA is a serotonin agonist and there is evidence to suggest that serotonin mediates antidiuretic hormone (ADH), MDMA-induced hyponatremia is due to syndrome of inappropriate hormone release, or SIADH. If SIADH is the pathophysiological mechanism suspected to be causing the hyponatremia, there may be a potential indication for a vasopressin receptor antagonist, such as tolvaptan or conivaptan. However, there is no evidence to support these agents in the treatment of MDMA-intoxication at this time. Another potential mechanism for MDMA-induced hyponatremia is that it is caused by excessive water intake. It is a common myth that drinking copious amounts of water will prevent MDMA-induced hyperthermia. Many patients begin drinking water once they present with excessive sweating or fever. Treatment of MDMA-induced hyponatremia should be conservative, as it often corrects itself as the intoxication resolves. Fluid status should be evaluated. Hypervolemic patients should be resuscitated with a crystalloid fluid. Hypotonic fluids should never be administered. Patients presenting with seizures or an altered mental status, suspected to be secondary to hyponatremia, should receive $3 \%$ hypertonic saline. The goal of sodium correction should be 1 to $2 \mathrm{mmol} / \mathrm{L} / \mathrm{hr}$, not to exceed the maximum correction rate of $10 \mathrm{mmol} /$ $\mathrm{L} / 24 \mathrm{hr}$. Once the patient is stabilized, fluid restriction is the preferred treatment. Patients presenting with altered mental status several hours after MDMA ingestion, commonly referred to as an "ecstasy hangover", ${ }^{10}$ may be experiencing symptomatic hyponatremia, rather than the effects of MDMA itself.

MDMA-induced seizures respond to boluses of midazolam. Boluses are recommended, as opposed to continuous infusions, for early recognition of regained consciousness or neurological damage. The cause of seizures in patients with MDMA intoxication may be multi-factorial. However, in many cases, the epileptic activity is secondary to hyponatremia. "Liquid ecstasy"," as "Fantasy" or gamma-hydroxybutyric acid (GHB), ${ }^{12}$ has no toxicological or chemical similarity to MDMA or ecstasy that was previously described. GHB was developed as an anesthetic drug in the 1960's that was removed from the market due to side effects. GHB is often used in combination with alcohol or other drugs. The most frequent signs and symptoms of GHB intoxication include headache, instability, disorientation, dysarthria, convulsions, emotional or behavior changes, including aggressiveness, nausea, vomiting, abdominal pain, dyspnea, chest pain and palpitations. Toxicological analyses for GHB are not routinely available in most laboratories for a definitive diagnosis to be made.

The mortality associated with GHB intoxications is generally low, as long as the patient is able to receive appropriate respiratory interventions. There is no known antidote to GHB intoxication, mainly due to its rapid absorption. The peak absorption time ranges from 23 minutes at $12.5 \mathrm{mg} / \mathrm{kg}$ to $45 \mathrm{minutes}$ at $50 \mathrm{mg} / \mathrm{kg}$. Fatty meals may delay this peak absorption time almost 3 -fold. Naloxone alone or in combination with flumazenil was administered in patients with GHB intoxication. However, no significant improvements in the level of consciousness resulted. Studies also show that the coma time does not vary, regardless of whether antidotes are administered or not. Activated charcoal has also been studied as a potential antidote in GHB intoxication. The American Academy of Clinical Toxicology states that there is no evidence supporting that activated charcoal improves the clinical outcome. However, it also states that there may be limited use in patients who have ingested large amounts of GHB and present for treatment within one hour of ingestion. Therapy should be based primarily on the preservation of the patient's respiratory status, often requiring intubation. Spice drugs, including $\mathrm{K}_{2}$, are synthetic cannabinoid derivatives. Like bath salts, synthetic Cannabinoids are legal highs sold over-the-counter in convenience stores under the guise of "not for human consumption." Synthetic cannabinoid formulations range from powders and liquids to smoking mixtures. Synthetic Cannabinoids are often smoked in hookah pipes. Spice drugs are typically a blend of plant and herbal ingredients sprayed with an active chemical agent, such as JHW-18, which is responsible for the toxicity of the synthetic Cannabinoids. There are various JHW compounds. The initials JHW refer to Dr. John W. Huffman who first synthesized analogs and metabolites of THC 
to study cannabinoid receptors. Synthetic Cannabinoids are agonists of the $\mathrm{CB}_{1}$ and $\mathrm{CB}_{2}$ receptors in the brain. The difference between synthetic Cannabinoids and THC are that the synthetic compounds are full agonists of the $\mathrm{CB}_{1}$ receptor and produce a more potent effect than THC, which is only a partial agonist. JHW-18, the most common of the synthetic Cannabinoids, has an affinity 5times greater on the $\mathrm{CB}_{1}$ receptor than THC. The more intense inhibition of the gammaaminobutyric acid neurotransmission in the brain by JWH-018 may be responsible for the symptoms of paranoia, agitation, anxiety, and dystonia. Synthetic Cannabinoids also have serotonin effects and are monoamine oxidase inhibitors, which may cause serotonin syndrome. Some formulations of spice also contain beta1-agonists, which cause hypertension, palpitations, tachycardia, anxiety, and irritability. There is no consistency in the composition of spice. Therefore, the signs and symptoms of toxicity are variable.

The American Association of Poison Control Centers reported that over 4,500 calls involving spice intoxication were made since 2010. Spice intoxicated patients typically present awake, but present with altered mental status and are unresponsive to painful stimuli. There is no antidote to spice intoxication. However, the symptoms are usually short acting and self-limited. Benzodiazepines are considered acceptable and effective treatment for the symptoms of agitation and restlessness. Diphenhydramine has been used with limited success in the reversal of dystonic reactions and muscular rigidity. It is recommended that electrolyte panels, electrocardiograms, urine drug screens, blood alcohol levels, and serum acetaminophen and salicylate levels be obtained in suspected synthetic cannabinoid toxicities. Synthetic stimulants, such as bath salts, MDMA or ecstasy, GHB, and spice, are becoming increasingly popular as substances of abuse. It is crucial for health care providers to recognize the symptoms and appropriately treat these patients. Urine toxicology tests should be ordered when an overdose of any agent is suspected. Positive drug screening assays not only identify causative agents, they link specific drug use and routes of administration with pharmacological effects. Many of these agents are marketed in combination and the chemical structures are continually changing. Often times, the urine tests will produce negative toxicology results because the tests are not updated as rapidly as the products are evolving or because the sample is not taken in time. Research has shown that the most common way to identify and diagnose an acute intoxication is through the patient interview. Patients or their family or friends will correctly identify the substance used in an overdose about $90 \%$ of the time, even if initial toxicology reports are inconclusive or negative. It is also important to notify local poison control centers of toxicities.

\section{Acknowledgements}

None.

\section{Conflict of interest}

Author declares that there is no conflict of interest.

\section{References}

1. Mas-Morey R, Visser MM, Winkelmolen L, et al. Clinical toxicology and management of intoxications with synthetic cathinones ("bath salts"). Journal of Pharmacy Practice. 2012:1-5.

2. Warrick BJ, Hill M, Hekman K, et al. A 9-State Analysis of Designer Stimulant, "Bath Salt", Hospital Visits Reported to Poison Control Centers. Ann Emerg Med. 2013;(20):101-108.

3. Jerry J, Collins G, Streem D. Synthetic legal intoxicating drugs: The emerging 'incense' and 'bath salt' phenomenon. Cleve Clin J Med. 2012;79(4):258-264.

4. Fass JA, Fass AD, Garcia AS. Synthetic cathiones (bath salts): legal status and patterns of abuse. The Annals of Pharmacotherapy. 2012;46(3):436-441.

5. Prosser JM, Nelson LS. The toxicology of bath salts: a review of synthetic cathinones. J Med Toxicol. 2011;8(1):33-42.

6. Wood KE. Exposure to bath salts and synthetic tetrahydrocannabinol from 2009 to 2012 in the United States. J Pediatr. 2013;163(1):213-216.

7. Spiller HA, Ryan ML, Weston RG, et al. Clinical experience with and analytical confirmation of "bath salts" and "legal highs" (synthetic cathinones) in the United States. Clin Toxicol (Phila). 2011;49(6):499-505.

8. Slominski A. A trip on "bath salts" is cheaper than meth or cocaine but much more dangerous. JAMA. 2012;308(23):2445-2447.

9. Traub SJ, Hoffman RS, Nelson LS. The Ecstasy Hangover: Hyponatremia due to 3,4-Methylenedioxymethamphetamine. J Urban Health. 2002;79(4):549-555.

10. Ben-Abraham R, Szold O, Rusick V, et al. "Ecstasy" intoxication: life-threatening manifestations and resuscitative measures in the intensive care setting. Eur J Emerg Med. 2003;10(4):309-313.

11. Galicia M, Nogue S, Miro O. Liquid ecstasy intoxication: clinical features of 505 consecutive emergency department patients. Emerg Med J. 2011;28(6):462-466.

12. Neijzen R, van Ardenne P, Sikma M, et al. Activated charcoal for GHB intoxication: An in vitro study. Eur J Pharm Sci. 2012;47(5):801-803. 